\title{
Approach to Assess Basic Deterministic Data and Model Form Uncertaint in Passive and Active Vibration Isolation
}

\author{
Roland Platz ${ }^{(凶)}$ \\ College of Engineering, Architectural Engineering, Penn State University, \\ University Park, PA 16802, USA \\ rxp5110@psu.edu \\ https://www.ae.psu.edu/index.aspx
}

\begin{abstract}
This contribution continues ongoing own research on uncertainty quantification in structural vibration isolation in early design stage by various deterministic and non-deterministic approaches. It takes into account one simple structural dynamic system example throughout the investigation: a one mass oscillator subject to passive and active vibration isolation. In this context, passive means that the vibration isolation only depends on preset inertia, damping, and stiffness properties. Active means that additional controlled forces enhance vibration isolation. The simple system allows a holistic, consistent and transparent look into mathematical modeling, numerical simulation, experimental test and uncertainty quantification for verification and validation. The oscillator represents fundamental structural dynamic behavior of machines, trusses, suspension legs etc. under variable mechanical loading. This contribution assesses basic experimental data and mathematical model form uncertainty in predicting the passive and enhanced vibration isolation after model calibration as the basis for further deterministic and nondeterministic uncertainty quantification measures. The prediction covers six different damping cases, three for passive and three for active configuration. A least squares minimization (LSM) enables calibrating multiple model parameters using different outcomes in time and in frequency domain from experimental observations. Its adequacy strongly depends on varied damping properties, especially in passive configuration.
\end{abstract}

Keywords: Vibration isolation - Passive and active damping Data uncertainty $\cdot$ Model form uncertainty

\section{Introduction to Data and Model Form Uncertainty}

Awareness and quantifying uncertainty in mathematical modeling, experimental test, and model verification and validation in early design stage are essential in structural dynamic application. The author recognizes the fact that, not rarely, uncertainty quantification approaches and documentation tend to lack transparency and comprehensibility, together with reluctance in consistently consolidating mathematic, stochastic and engineering terminology. This makes it often

(C) The Author(s) 2021

P. F. Pelz and P. Groche (Eds.): ICUME 2021, LNME, pp. 208-223, 2021.

https://doi.org/10.1007/978-3-030-77256-7_17 
impractical, or too difficult and time consuming to transfer and apply uncertainty quantification measures to common and real engineering problems.

Generally, literature subdivides uncertainty in aleatoric and epistemic uncertainty, [19,20,24]. Aleatoric uncertainty is irreducible and mostly characterized by probabilistic distribution functions. It is presumed intrinsic randomness of an outcome. Epistemic uncertainty is reducible and occurs due to lack of knowledge, or insufficient of incomplete data or models, [2]. Data includes model parameters and state variables, a model determines the functional relation of data. The German Collaborative Research Center SFB 805 "Control of Uncertainty in Load-Carrying Structures in Mechanical Engineering", which funds this work, distinguishes between data and model form uncertainty. Currently, the SFB 805 discusses a third characteristic, structure uncertainty. Data uncertainty may appear as stochastic uncertainty or incertitude, [11]. In case of stochastic uncertainty, probabilistic measures like BAYES-inferred MONTE CARLO simulations process known or assumed distribution functions of data. In case of incertitude, non-probabilistic measures like FUZZY and interval analysis process membership functions and intervals. Ignorance of uncertainty prevails if neither stochastic uncertainty nor incertitude are taken into account.

Model form uncertainty expresses unknown, incomplete, inadequate or unreasonable functional relations between the model input and output, model parameters and state variables when compared to observations from real experimental test. The scope and complexity of the model also have an impact on the severity of the uncertainty. The dilemma the designer encounters in early stage design, before calibration, verification and validation processes start, is the extent of uncertainty. The works $[5,7]$ introduce a general relation between a real observation from experiments and a mathematical model to identify model form uncertainty. An observation reflects the measured physical outcome, mostly as states like forces, displacement, accelerations etc. A model must reflect the same outcome, which depends on data and the chosen functional relations. The difference between the outcome of the observation and the model is a combination of quantified model deviation and measurement uncertainty, including noise. Deterministic or non-deterministic approaches estimate the deviation, for example as a discrepancy function.

As a first step, prior to develop discrepancy functions, this paper quantifies basic deviations between experimental and numerical simulated outcomes of a one mass oscillator's passive and active vibration isolation capability. The investigated one mass oscillator is equipped with a velocity feedback controller that realizes passive and active damping. Considering this particular structural dynamic example, Platz et al. so far investigated the influence of data uncertainty on the vibrational behavior in frequency domain by numerical simulations in [16-18] in. Lenz et al. [8] conducted experimental investigations with regard to data uncertainty of the same system, introduced in [15]. The investigations covered data uncertainty in frequency domain.

The current paper looks upon experimental data and model form uncertainty in frequency and in time domain. First, it presents the derivation of 
the analytical excitation and response models. Second, the author explains the test setup, followed by discussing measured data, resp. measuring uncertainty. Third, remaining deviations between experimental observation and predictions by mathematical models after calibrating selected model parameters disclose model form uncertainty.

\section{Analytical Model}

A one mass oscillator is the most simple representation of a vibrating rigid body system to describe linear passive and active vibration isolation for many structural dynamic systems. For example, it is often used for first numerical estimation of a driving car's vertical dynamic behavior, [23], Fig. 1.

a)

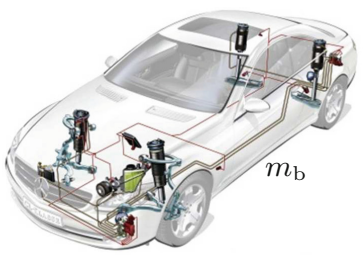

b)

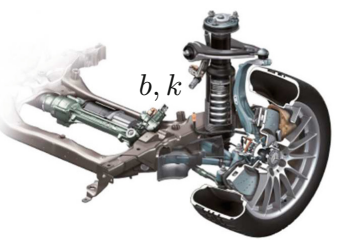

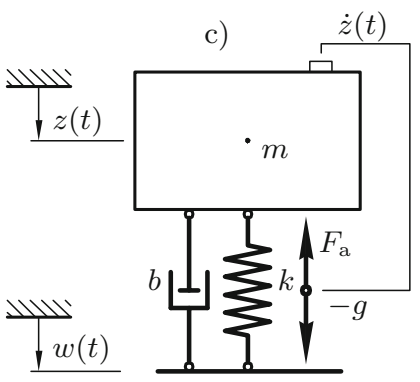

Fig. 1. Derivation of a one mass oscillator, a) automobile (C)Auto Reporter/Mercedes Benz) with total mass $m_{\mathrm{b}}, \mathrm{b}$ ) front suspension leg (Mercedes Benz) with damping $b$ and stiffness $k$, mass of suspension leg neglected, c) one mass oscillator model with position excitation $w(t)$ of a massless base point, and active vibration isolation by active velocity feedback control force $F_{\mathrm{a}}=-g \dot{z}$

The analytical mathematical model represents only one fourth of the car's chassis and one suspension leg, with the chassis mass $m$ and the suspension leg's damping and stiffness properties: damping coefficient $b$ and the stiffness $k$ in passive configuration. For active configuration, a velocity feedback with gain $g$ is added to provide an active force, Fig. 1c. The absolute vertical displacement $z(t)$ of the mass and the base point displacement excitation function $w(t)$ depend on time $t$. The base point is assumed without inertia, represented only by a horizontal line. For example, $w(t)$ represents driving on an uneven bumpy road.

The inhomogeneous differential equation of motion for mass $m$

$$
\ddot{z}(t)+\left[2 D_{\mathrm{p}} \omega_{0}+\frac{g}{m}\right] \dot{z}(t)+\omega_{0}^{2} z(t)=2 D_{\mathrm{p}} \omega_{0} \dot{w}(t)+\omega_{0}^{2} w(t)=\omega_{0}^{2} r(t)
$$

of the one mass oscillator includes the damping ratio $D_{\mathrm{p}}$ from passive damping, $0<D_{\mathrm{p}}<1$, as well as the angular eigenfrequency $\omega_{0}$

$$
2 D_{\mathrm{p}} \omega_{0}=\frac{b}{m}, \text { and } \omega_{0}^{2}=\frac{k}{m} \text {. }
$$


The term $r(t)$ in (1) is the general expression of the excitation function multiplied by $\omega_{0}^{2}$. In this particular case it is the linear combination of a damper-spring base point excitation, [6].

\subsection{Vibration Excitation and Responses}

With $w(t) \neq 0$ and $\dot{w}(t) \neq 0$ in $(1)$, the solution $z(t)$ is a linear combination of free and forced vibration responses in time domain. In frequency domain, the forced vibrational response depends on the magnitude and frequency content of the excitation source. The base point excitation $w(t)$ triggers $z(t)$. It is assumed that $z(t)$ is the response of a step function resulting from an initial impulse applied on the rigid frame, Sect. 3 .

\subsection{Time Domain}

The unit step function

$$
\sigma\left(t-t_{0}\right)= \begin{cases}0 & \text { for } t<t_{0} \\ 1 / 2 & \text { for } t=t_{0} \\ 1 & \text { for } t>t_{0}\end{cases}
$$

is an ideal excitation model of the sudden change from the state 0 for $t<t_{0}$ to state 1 for $t>t_{0}$ before or after a certain point of time $t_{0}$. The dynamic system's vibration response

$$
z(t)=r_{0}\left\{1-e^{-D \omega_{0} t}\left[\cos \omega_{D} t-D \frac{\omega_{0}}{\omega_{D}} \sin \omega_{D} t\right]\right\}
$$

for $t>t_{0}$ is the sum of the system's free vibration response solution $z_{\mathrm{h}}(t)$ of the homogeneous equation of motion (1), with initial conditions $w\left(t_{0}\right)=0$ and $\dot{w}\left(t_{0}\right)=0$, and the forced vibration response

$$
r_{0}=\frac{1}{\omega_{0}} 2 D_{\mathrm{p}} \dot{w}_{0}+w_{0} .
$$

(5) is the assumptive particular solution $z_{\mathrm{ih}}(t)$ of the inhomogeneous equation of motion (1) for $w_{0}=w\left(T_{\mathrm{i}}\right) \neq 0$ and $\dot{w}_{0}=\dot{w}\left(T_{\mathrm{i}}\right) \neq 0$, when the impulse ends at time $T_{\mathrm{i}},[6]$, Sect. 4.1 .

\subsection{Frequency Domain}

The frequency content of the excitation source determines the amplitude frequency and phase frequency response. (1) is transferred into frequency domain

$$
\left\{-\Omega^{2}+i \Omega\left(2 D_{\mathrm{p}} \omega_{0}+\frac{g}{m}\right)+\omega_{0}^{2}\right\} \underline{\underline{z}}_{\mathrm{ih}} e^{i \Omega t}=\left\{i \Omega 2 D_{\mathrm{p}} \omega_{0}+\omega_{0}^{2}\right\} \underline{\widehat{w}} e^{i \Omega t}
$$


by adding the sine term $i \sin (\Omega t+\varphi)$ as a complex extension, and by using exponential form with the constant and complex excitation amplitude magnitude $\underline{\widehat{w}}$, and the complex response amplitude magnitude $\underline{\underline{z}}_{\mathrm{ih}}$. The complex vibrational displacement response of the mass $m$

$$
\underline{\widehat{z}}_{\mathrm{ih}}=\frac{i \Omega 2 D_{\mathrm{p}} \omega_{0}+\omega_{0}^{2}}{-\Omega^{2}+i \Omega\left(2 D_{\mathrm{p}} \omega_{0}+\frac{g}{m}\right)+\omega_{0}^{2}} \underline{\widehat{w}},
$$

using

$$
\zeta=\frac{\Omega}{m \omega_{0}^{2}} \text { and } \eta=\frac{\Omega}{\omega_{0}},
$$

results in the complex magnifying function

$$
\underline{V}(\eta)=\frac{\widehat{\underline{z}}_{\mathrm{ih}}}{\underline{\widehat{w}}}=\frac{i 2 D_{\mathrm{p}} \eta+1}{1-\eta^{2}+i\left(2 D_{\mathrm{p}} \eta+g \zeta\right)},
$$

leading to the amplitude response

$$
|\underline{V}(\eta)|=\sqrt{\frac{\left(2 D_{\mathrm{p}} \eta\right)^{2}+1}{\left(1-\eta^{2}\right)^{2}+\left(2 D_{\mathrm{p}} \eta+g \zeta\right)^{2}}}
$$

and phase response

$$
\psi(\eta)=\arctan \frac{-2 D_{\mathrm{p}} \eta^{3}-g \zeta}{1-\eta^{2}+\left(2 D_{\mathrm{p}} \eta\right)^{2}+2 D_{\mathrm{p}} \eta g \zeta} .
$$

\section{Experimental Test Setup}

Figure 2 explains the real test setup concept, with the one mass oscillator model embedded in a frame with a relatively heavy mass $m_{\mathrm{f}} \gg m$ as the real test setup concept. It contains the physical and real representation of the base point for experimental testing. The frame is excited by the force $F(t)$ due to an impulse using a modal hammer; it is connected to the ground via an elastic support with relatively low damping $b_{\mathrm{f}} \ll b$ and low stiffness $k_{\mathrm{f}} \ll k$. These properties lead to a quasi-static dynamic response of the frame after the impulse, with a relatively low first eigenfrequency $\omega_{0, \mathrm{f}} \approx 2 \pi 1 / \mathrm{s} \ll \omega_{0}$, compared to the first eigenfrequency $\omega_{0}$ of the mass $m$. It is fair to assume that the forced vibration response $z(t)$ is the result of an assumed one mass oscillator.

The virtual rigid frame model with mass $m_{\mathrm{f}}$ in Fig. 2 is fixed by an idealized gliding support assumed to have no friction perpendicular to the $z$-direction. The support permits a frame movement only in $z$-direction. The frame is constrained by an idealized damper with the damping coefficient $b_{\mathrm{f}}$ and a spring with the stiffness $k_{\mathrm{f}}$ in $z$-direction. The frame suspends from a rigid mount via elastic straps vertical to the $z$-direction, allowing low frequency pendulum motion of the frame in $z$-direction, Fig. 3. This motion is the translational absolute excitation displacement $w(t)$ in $z$-direction, when the frame is excited by a hammer impulse. Figure 3 shows the real test setup. 


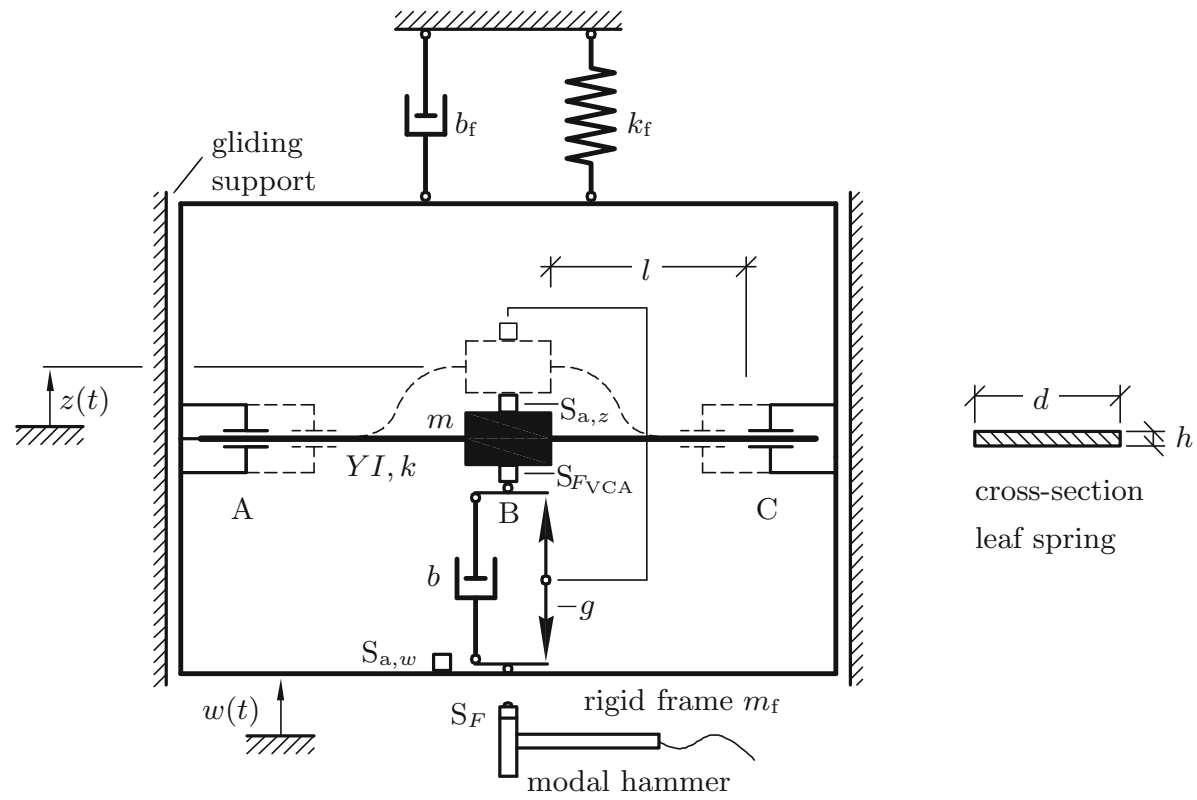

Fig. 2. One mass oscillator - schematic diagram of real test set up
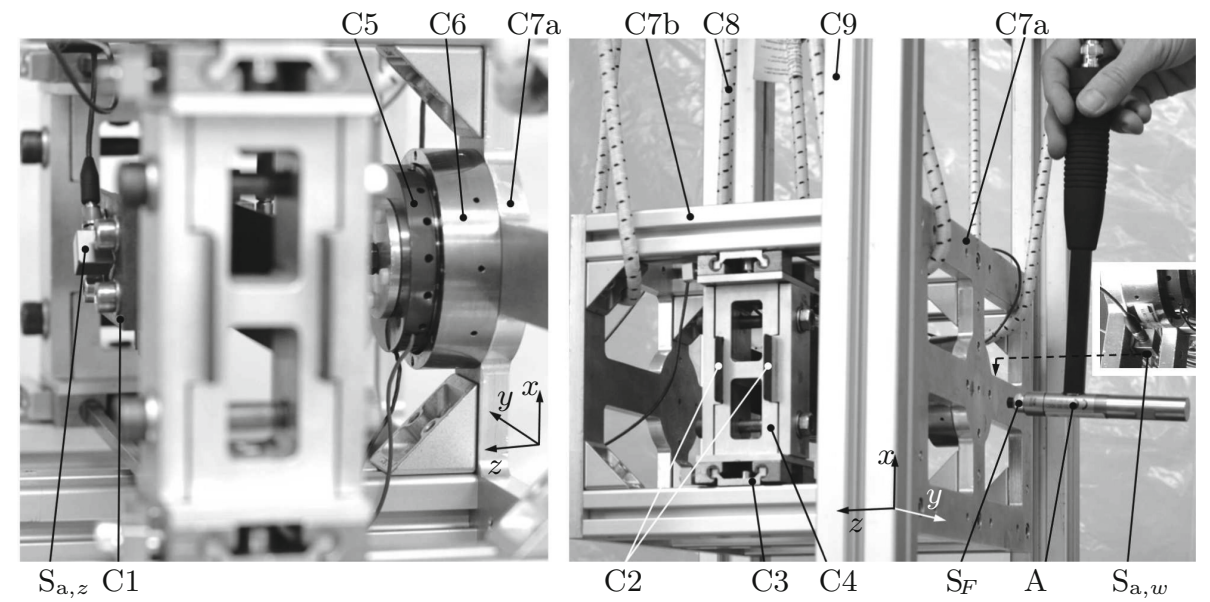

Fig. 3. Physical test setup - left: assembly of leaf spring and VCA; right: hammer impulse on frame; the components are: acceleration sensor $\mathrm{S}_{\mathrm{a}, z}$ attached to the oscillating mass $\mathrm{C} 1$, two leaf springs $\mathrm{C} 2$ with partial stiffness $k / 2$ on each side of $\mathrm{C} 1$, glide support C3, fixed leaf spring support C4, VCA coil support/holder C5, VCA stator, magnet outer ring $\mathrm{C} 6$, front/side structure of rigid frame $\mathrm{C} 7 \mathrm{a} / \mathrm{b}$ with total mass $m_{\mathrm{f}}$, elastic strap C8, mount C9 to suspend the frame with elastic straps, acceleration sensor $\mathrm{S}_{\mathrm{a}, w}$ (hidden behind the frame) on the frame mass $m_{\mathrm{f}}$, and force sensor $\mathrm{S}_{F}$ measuring the impulse force from the model hammer $\mathrm{A}$ 
The frame in Figs. 2 and 3 retains two supports that fix a leaf spring at its ends at $\mathrm{A}$ and $\mathrm{C}$, with the effective bending length $l$ on sides $\mathrm{A}-\mathrm{B}$ and $\mathrm{B}-\mathrm{C}$, and with the rigid mass $m$ in the center position at $\mathrm{B}$. The leaf spring is the practical realization of the spring elements in Fig. 1c. Its cross section area is $d h$, with the cross section width $d$ and height $h$; its stiffness $k$ is a function of the bending stiffness $E I ; E$ is the elastic or YounG's modulus of the leaf spring made from carbon fiber reinforced polymer (CFRP), $I$ is the area moment of inertia. The two supports at $\mathrm{A}$ and $\mathrm{C}$ are adjustable along $l$ to tune the leaf spring's bending deflection, and eventually its effective stiffness $k$. A voice coil actuator (VCA) provides the passive and active damping forces $F_{b}$ and $F_{\mathrm{a}}$. The VCA's electromotive force

$$
F_{\mathrm{S}_{\mathrm{VCA}}}=F_{b}+F_{\mathrm{a}}=b[\dot{z}(t)-\dot{w}(t)]-g \dot{z}(t)
$$

is detected via the sensor $\mathrm{S}_{\mathrm{VCA}}$, Fig. 3. Two acceleration sensors $\mathrm{S}_{\mathrm{a}, z}$ and $\mathrm{S}_{\mathrm{a}, w}$ measure the absolute accelerations $\ddot{z}(t)$ and $\ddot{w}(t)$ of the mass and the frame. The absolute accelerations are transformed into absolute velocities $\dot{z}(t)$ and $\dot{w}(t)$ by numerical integration in the SIMULINK-DSPACE ${ }^{\mathrm{TM}}$ environment. The masses of the sensors $\mathrm{S}_{\mathrm{a}, z}, \mathrm{~S}_{F_{\mathrm{VCA}}}$, and of the leaf spring, are considered parts of the oscillating mass $m$. Gravitational forces are neglected, the directions of $z(t)$ and $w(t)$ of the test rig are perpendicular to gravitation.

\section{Experimental Models}

\subsection{Excitation}

The frame's vibrational displacement response

$$
w(t)=\frac{\check{F}\left(t_{0}\right)}{m_{\mathrm{f}} \omega_{\mathrm{D}, \mathrm{f}}} e^{-D_{\mathrm{f}} \omega_{0, \mathrm{f}} t} \sin \omega_{\mathrm{D}, \mathrm{f}} t
$$

is the result from the impulse force

$$
\check{F}=\int_{t_{1}}^{t_{2}} F(t) \mathrm{d} t
$$

as the integral of the force function

$$
F(t)= \begin{cases}\frac{\widehat{F}}{2}\left[\sin \left(\Omega_{\mathrm{i}} t-\frac{\pi}{2}\right)+1\right] & \text { between } 0 \leq t \leq T_{\mathrm{i}} \\ 0 & \text { for } t>T_{\mathrm{i}}\end{cases}
$$

assumed form experiments, with the peak force $\widehat{F}$ within a short time period between 0 and $T_{\mathrm{i}}$. The response (13) is valid for low damping $0<D_{\mathrm{f}}<1$, $[6,10]$. When the impulse ends at $t=T_{\mathrm{i}}$, the response (13) reaches a value that is approximated as the frame's effective displacement response step hight $w_{0}=w\left(T_{\mathrm{i}}\right)$, which is used to determine the step hight $r_{0}$ in (5) to calculate the step response $z(t)$ in (4). 


\subsection{Stiffness}

The stiffness

$$
k^{*}=\frac{12 E I}{l^{3}}
$$

derives from one leaf spring's flexural bending stiffness $E I$ with respect to the length $l$ between $\mathrm{A}$ and B, and between B and C, Fig. 2. The effective stiffness with four leaf springs becomes $k=4 \cdot k^{*}$. It is the sum of four added stiffnesses, linearity assumed, with two leaf springs at each side $\mathrm{A}-\mathrm{B}$ and $\mathrm{B}-\mathrm{C}$ of the mass $m$.

\subsection{Damping}

The VCA provides the passive damping and active gain forces $F_{b}$ and $F_{\mathrm{a}}(12)$ for passive and active vibration isolation, [15]. The LORENTz force

$$
F_{\mathrm{VCA}}=r l_{\mathrm{c}} i \cdot B=\Psi i=b(\dot{z}-\dot{w})+g \dot{z}
$$

is expressed by the force constant $\Psi$, length of the coil $l_{\mathrm{c}}$ and the ratio $r$ of the effective coil length, the magnetic flux density $B$, and the electrical current $i$, if $B$ and $i$ are perpendicular to each other. The VCA's driving electrical power is

$$
P=u_{\mathrm{VCA}} i=F_{\mathrm{VCA}} v
$$

and equivalent to the driving mechanical power $F_{\mathrm{VCA}} v$. The driving voltage $u_{\mathrm{VCA}}=\Psi v$ initiates the electromotive force $F_{\mathrm{VCA}}$. The VCA's properties inductance $L$, Ohmic resistance $R$, and force constant $\Psi$ lead to the control voltage

$$
u=\Psi v+\frac{\mathrm{d} i}{\mathrm{~d} t} L+i R .
$$

Eventually, the applied control voltage

$$
u=\left\{\Psi(\dot{z}-\dot{w})+\frac{\mathrm{d}}{\mathrm{d} t}\left[\frac{1}{\Psi}(b(\dot{z}-\dot{w})+g \dot{z})\right] L+\frac{1}{\Psi}(b(\dot{z}-\dot{w})+g \dot{z}) R\right\}
$$

depends on the relative velocity $\dot{z}-\dot{w}$ between the frame and the mass to provide the passive damping force $F_{b}$, and on the absolute velocity $\dot{z}$ of the mass to provide the active force $F_{\mathrm{a}}$. It depends directly on $b$ and $g$.

\subsection{Frequency Response Estimation and Coherence}

The amplitude and phase estimation

$$
|V(\Omega)|=\left|H_{2}(\Omega)\right|=\left|\frac{S_{\tilde{z}, \tilde{z}}(\Omega)}{S_{\tilde{z}, \tilde{w}}(\Omega)}\right|, \quad \psi(\Omega)=\angle H_{2}(\Omega)
$$

process the auto-power and cross-power spectral densities $S_{\tilde{\ddot{z}}, \tilde{z}}(\Omega)$ and $S_{\tilde{z}, \tilde{\ddot{w}}}(\Omega)$ from the measured mass acceleration response $\tilde{\ddot{z}}(t)$, and from the frame acceleration excitation $\tilde{\ddot{w}}(t)$ from hammer excitation, averaged 5 -times. The signals 
take into account normal perturbations from inexact and manual handling of the impulse hammer during averaging, marked by the tilde . The well known estimator $H_{2}(\Omega)$ leads to relatively small response errors in resonance compared to higher errors for anti-resonances. The coherence

$$
\gamma^{2}(\Omega)=\frac{\left|S_{\tilde{z}, \tilde{\tilde{w}}}(\Omega)\right|^{2}}{S_{\tilde{z}, \tilde{z}}(\Omega) S_{\tilde{\tilde{w}}, \tilde{\tilde{w}}}(\Omega)}, \quad 0 \leq \gamma^{2}(\Omega) \leq 1
$$

determines the quality of the experimental signals, with full correlation, resp. highest estimation quality at $\gamma^{2}(\Omega)=1$, and no correlation, resp. lowest estimation quality at $\gamma^{2}(\Omega)=0$ between the excitation and response signals.

\section{Deterministic Uncertainty Measures}

\subsection{Measurement and Data Uncertainty}

The validity of the sensor sensitivity is checked by exciting the mass $m 10 \mathrm{~Hz}$ with a $u=2 \mathrm{~V}$ amplitude input from the VCA, and measuring the force and acceleration outputs $F_{\mathrm{S}_{\mathrm{VCA}}}$ and $a_{\mathrm{S}_{\mathrm{a}, z}}$. The two outputs are converted back to voltage signals $u_{\mathrm{S}_{\mathrm{VCA}}}$ and $u_{\mathrm{S}_{\mathrm{a}, z}}$ via their sensitivities $0.01124 \mathrm{~V} / \mathrm{N}$ and $102 \mathrm{~V} /\left(\mathrm{m} / S^{2}\right)$, given by the manufacturers [13,14], and compared to the measured voltage outputs $u_{\mathrm{Osc}_{\mathrm{VCA}}}$ and $u_{\mathrm{Osc}_{a}}$ from a parallel connected oscilloscope. The VCA gets its defined input signal from a signal generator.

For the validity of the measurement chain, the modal hammer hits a calibrated $1 \mathrm{~kg}$ mass hold in hand. The force signal excitation peak $F_{\mathrm{S}_{F}}$ of the hammer impulse is the input, the acceleration response peak $a_{\mathrm{S}_{\mathrm{a}, z}}$ is the output, both are low pass filtered $500 \mathrm{~Hz}$ by an analogue filter. A DSPACE ${ }^{\mathrm{TM}}$ and MATLAB ${ }^{\mathrm{TM}}$ real time controller processes the signals. In addition, the power spectra density estimator $H_{2}(\Omega)(21)$ determines the measured frequency response between $0 \leq \Omega / 2 \pi \leq 200 \mathrm{~Hz}$. If measurement uncertainty is absent, $H_{2}(\Omega)$ must be $0 \mathrm{db}$ over the entire frequency range.

For checking the stiffness reproducibility, the leaf-spring length $l=0.08 \mathrm{~m}$ in Fig. 2 leads to the lowest possible stiffness $k=4 k^{*}=25,788 \mathrm{~N} / \mathrm{m}$ for the design according to (16), with the elastic modulus $E=62 \cdot 10^{9} \mathrm{~N} / \mathrm{m}^{2}$ for CFRP, and the area moment of inertia $I$ from $d=4 \cdot 10^{-2}$ and $h=0.11 \cdot 10^{-2} \mathrm{~m}$. After measuring the frequency response (21), the screws in the fixed leaf spring support are loosened and tightened three times to again adjust the specified length $l$, after manually shaking the assembly for few moments. This procedure is repeated three times to see if there are any different resonance peaks according to (2) with changing stiffness from (21), after mounting and dismounting the leaf spring support.

Table 1 quantifies the measurement uncertainty after the calibration of sensors, measurement chain, and reproducibility of stiffness adjustment. With $2 \%$ and $10 \%$, the uncertainty in force and acceleration signals remain below $\pm 15 \%$ and close to $\pm 10 \%$ of the uncertainty margins given by the sensor manufacturer, [12-14]. The measurement chain provides low uncertainty with $\approx 3 \%$ deviation from the expected $1 \mathrm{~kg}$ and $\approx-0.18 \mathrm{~dB}$ from the expected $0 \mathrm{~dB}$. The reproducibility check from assembling discloses a stiffness deviation of $\approx 6 \%$. 
Table 1. Measurement uncertainty from sensors sensitivity, measurement chain, and stiffness reproducibility affecting the eigenfrequency

\begin{tabular}{l|l|l|l|l|l}
\hline & \multicolumn{3}{|l|}{ States } & Model parameter $k$ \\
\hline & Force & Acceleration & \multicolumn{2}{l}{ Measurement chain } & Eigenfrequency $(2)$ \\
& $u_{\mathrm{Osc} \mathrm{VCA}} / u_{\mathrm{S}_{\mathrm{VCA}}}$ & $u_{\mathrm{Osc}_{a}} / u_{\mathrm{S}_{a}}$ & $F_{\mathrm{S}_{F}} / a_{\mathrm{S}, z}$ & $\left|H_{2}\right|$ & $\omega_{\min } / \omega_{\max }$ \\
$\operatorname{dev.}$ & $\approx 2 \%$ & $\approx 10 \%$ & $\approx 1.03 \mathrm{~kg}$ & $\approx-0.18 \mathrm{~dB}$ & $\approx 6 \%$ \\
\hline
\end{tabular}

\subsection{Model Form Uncertainty}

The relevant vibration isolation outcomes: excitation hammer force (15), frame and mass accelerations from (13) and (4), as well as phase (10) and amplitude (11) from experiments and models after calibration in time domain, are measured by experiments and calculated from mathematical models. The objective function

$$
\min _{\theta \in \mathbb{R}} \frac{1}{N} \sum_{n}^{N} \sum_{p}^{P} \frac{\left\{y_{p}\left(X_{n}\right)-v_{p}\left(X_{n}, \theta\right)\right\}^{2}}{\max \left|y_{p}\left(X_{n}\right)\right|^{2}}
$$

for model calibration, or, respectively, model updating is a least squares minimization (LSM). It uses $P$ observation outcomes $y_{p}\left(X_{n}\right)$ and $P$ predicted model outcomes $v_{p}\left(X_{n}, \theta\right)$ with control parameter $X_{n}=\left[t_{n}, f_{n}\right]$ as discrete time and frequency elements, and calibration parameters $\theta=[k, b, g]$. In this work, the outcomes may appear in time and/or in frequency domain, leading to $N=4096$ time samples and/or $N=2048$ frequency samples. In this example, the control parameter $X_{n}$ is frequency. The number of outcomes is $P=2$, only $|V|$ and $\psi$ are used in (23). LSM is conducted by the particle swarm optimization (PSO) algorithm [3]; it reduces the risks of converging the LSM to a local minimum, [4]. All other relevant and remaining model parameters: oscillating mass $m=0.9249$, frame mass $m_{\mathrm{f}}=9.33 \mathrm{~kg}$, stiffness $k_{\mathrm{f}}=722 \mathrm{~N} / \mathrm{m}$, and damping $b_{\mathrm{f}}=10 \mathrm{Ns} / \mathrm{m}$, as well as state variables: excitation force amplitude $\widehat{F}=135 \mathrm{~N}$ and impulse time $T_{\mathrm{i}}=5 \cdot 10^{-3} \mathrm{~s}$, are assumed constant. However, they are potential candidates as calibration parameters in succeeding investigations.

Figure 4 displays phase and frequency responses as the outcomes for LSM for three different passive damping cases a) to c) and three different active damping cases $\mathrm{d}$ ) to $\mathrm{f}$ ), Table 2 . The coherence $\gamma^{2}(f)(22)$ evaluates the quality of the experimental observations. The objective function $(23)$ uses $y_{1}(f)=\psi^{\mathrm{e}}(f)$ and $y_{2}(f)=\left|V^{\mathrm{e}}(f)\right|$ from experimental observation, and $v_{1}(f, k, b, g)=\psi^{\mathrm{m}}(f)$ and $v_{2}(f, k, b, g)=\left|V^{\mathrm{m}}(f)\right|$ from numerical simulation using the models after parameter calibration. Figure 4 also shows the model outcome from the initially guessed parameter $k$ after adjusting the leaf spring length $l$ in (16), as well as $b$, and $g$ for the VCA's control voltage $u(20)$ used to specify the test rig properties. Further, it shows the deviation between the calibrated and measured outcomes. Table 2 lists the initially chosen and calibrated parameters $k, b$, and $g$, along with the deviations and remaining LSM-values after calibration. The VCA's 
given parameters are inductance $L=0,003 \mathrm{Vs} / \mathrm{A}$, resistance $R=4.8 \mathrm{~V} / \mathrm{A}$, and force constant $\Psi=17.5 \mathrm{~V} / \mathrm{A},[1]$.

Table 2. Model parameters from initial guess, after calibration, values of least square minimization (LSM), deviations between $k, b$, and $g$ from initial guess and calibration

\begin{tabular}{|c|c|c|c|c|c|}
\hline & & $\begin{array}{l}k \\
\text { in } \mathrm{N} / \mathrm{m}\end{array}$ & $\begin{array}{l}b \\
\text { in } \mathrm{Ns} / \mathrm{m}\end{array}$ & $\begin{array}{l}g \\
\text { in } \mathrm{Ns} / \mathrm{m}\end{array}$ & $\begin{array}{l}\text { LSM } \\
-\end{array}$ \\
\hline \multirow{3}{*}{ a) } & Initial guess & 32,583 & 41.66 & 0 & \multirow{3}{*}{0.0069} \\
\hline & Calibrated & 32,682 & 41.22 & 0 & \\
\hline & Deviation in $\%$ & 0.30 & -1.06 & - & \\
\hline \multirow{3}{*}{ b) } & Initial guess & 32,583 & 69.44 & 0 & \multirow{3}{*}{0.0045} \\
\hline & Calibrated & 36,639 & 63.87 & 0 & \\
\hline & Deviation in $\%$ & 12.44 & -8.01 & - & \\
\hline \multirow{3}{*}{ c) } & Initial guess & 32,583 & 97.21 & 0 & \multirow{3}{*}{0.0125} \\
\hline & Calibrated & 42,649 & 87.71 & 0 & \\
\hline & Deviation in $\%$ & 30.89 & -9.77 & - & \\
\hline \multirow{3}{*}{ d) } & Initial guess & 32,583 & 18.30 & 22.00 & \multirow{3}{*}{0.0026} \\
\hline & Calibrated & 32,239 & 23.68 & 11.00 & \\
\hline & Deviation in $\%$ & -1.06 & 29.43 & -50.00 & \\
\hline \multirow{3}{*}{ e) } & Initial guess & 32,583 & 18.30 & 46.00 & \multirow{3}{*}{0.0013} \\
\hline & Calibrated & 33,926 & 11.95 & 29.70 & \\
\hline & Deviation in $\%$ & 4.12 & -34.66 & -35.44 & \\
\hline \multirow{3}{*}{ f) } & Initial guess & 32,583 & 18.30 & 68.00 & \multirow{3}{*}{0.0031} \\
\hline & Calibrated & 35,219 & 9.14 & 43.62 & \\
\hline & Deviation in $\%$ & 8.09 & -50.00 & -35.86 & \\
\hline
\end{tabular}

Figure 5 shows the outcomes: excitation hammer force, frame and mass accelerations. They are not used by the objective function (23). The calibrated parameters $k, b$, and $g$ do not affect the deviations between guessed vs. measured forces $F(t)$, nor do they affect the frame acceleration $\ddot{w}(t)$. The deviations are caused by multiple nonidentical experimental trials. The deviations between calibrated vs. measured oscillating mass acceleration $\ddot{z}(t)$, however, are affected by the calibration parameters.

First of all, it is worth noting that the results in Fig. 4 confirm the improved vibration isolation effect by active measures in the cases d) to f). In both experimental and numerical simulation, a strong vibration isolation effect for frequencies higher than resonance frequencies is present with increasing damping, compared to the passive cases a) to c). Second, increasing passive and active damping shows that the calibrated model parameters $k, b$ and $g$ deviate significantly from the initially guessed parameters used in the experiments, Table 2. Specifically, the increasing damping in the passive configuration leads to less adequate 
a) Phase response, $\ddot{z} / \ddot{w}$

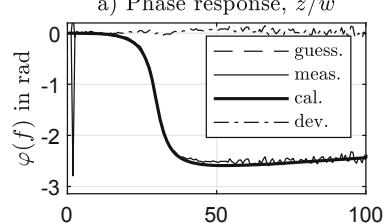

a) Amplitude response, $\ddot{z} / \ddot{w}$

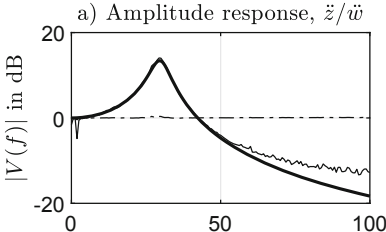

a) Coherence, $\ddot{z} / \ddot{w}$

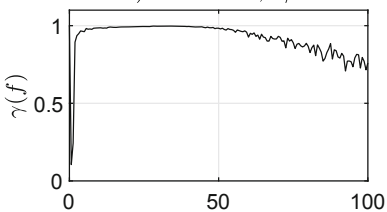

d) Phase response, $\ddot{z} / \ddot{w}$
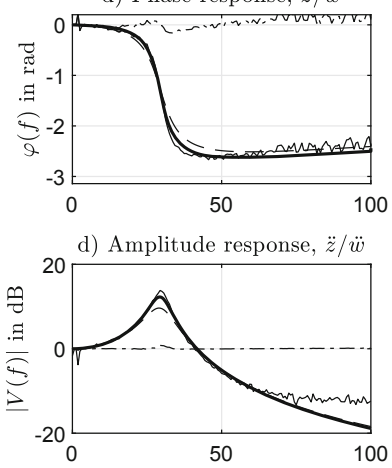

d) Coherence, $\ddot{z} / \ddot{w}$

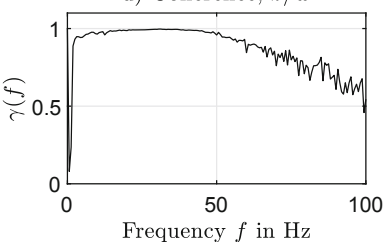

b) Phase response, $\ddot{z} / \ddot{w}$

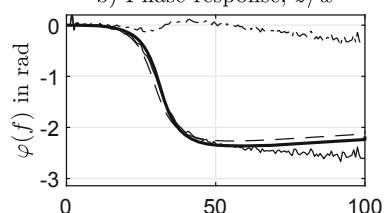

b) Amplitud

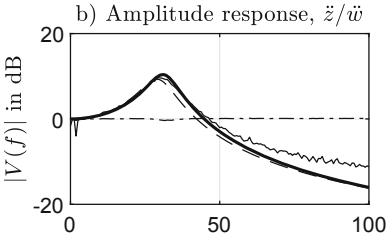

b) Coherence, $\ddot{z} / \ddot{w}$

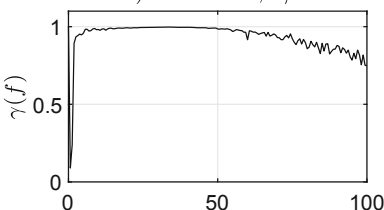

e) Phase response, $\ddot{z} / \ddot{w}$
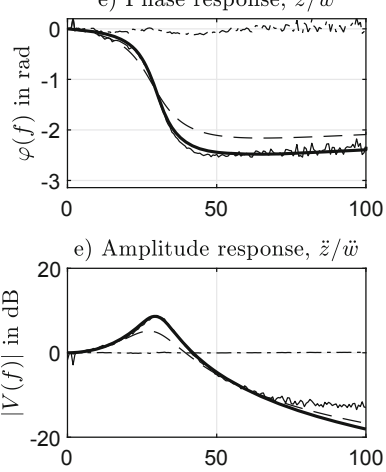

e) Coherence, $\ddot{z} / \ddot{w}$

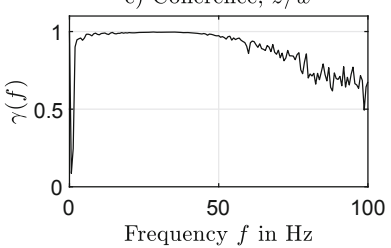

c) Phase response, $\ddot{z} / \ddot{w}$
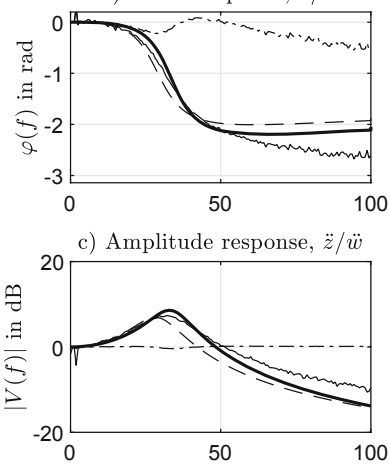

c) Coherence, $\ddot{z} / \ddot{w}$

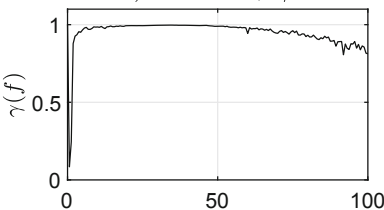

f) Phase response, $\ddot{z} / \ddot{w}$
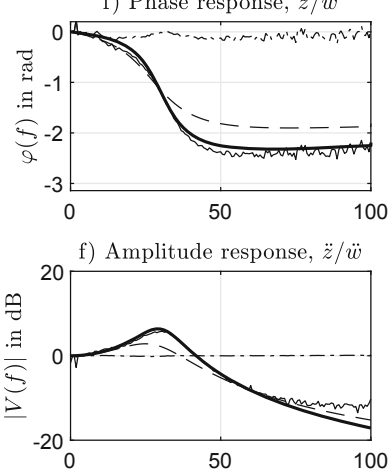

f) Coherence, $\ddot{z} / \ddot{w}$

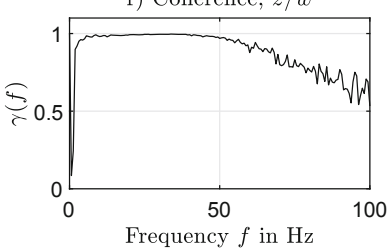

Fig. 4. Outcome, initially guessed from models, from measurement, from calibration, deviations between measurement and calibration of phase $\psi(f)$ and amplitude $|V(f)|$ frequency responses, and choherence $\gamma^{2}(f)$ in frequency domain for six damping cases a) to d), Table 2 

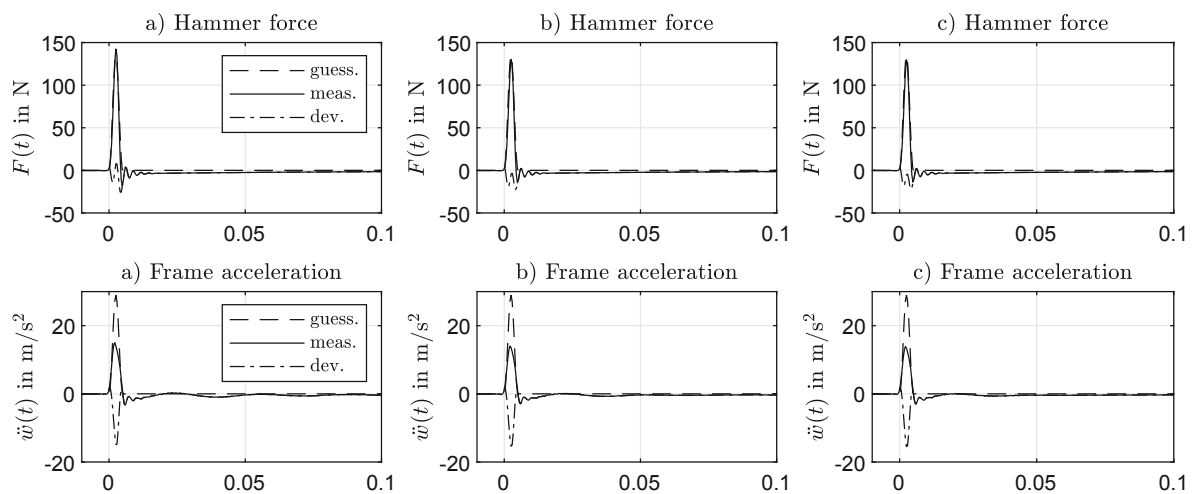

b) Frame acceleration

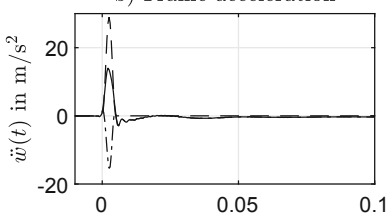

c) Frame acceleration

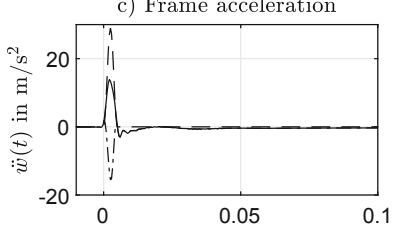

a) Mass acceleration

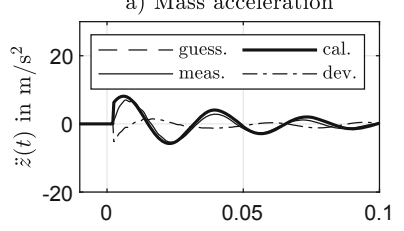

b) Mass acceleration

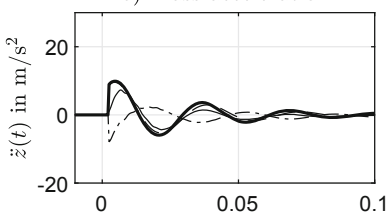

c) Mass acceleration

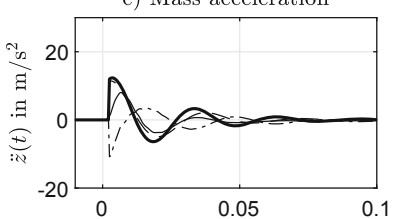

e) Hammer force
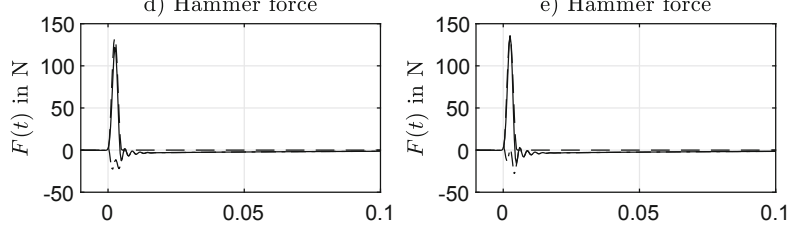

f) Hammer force

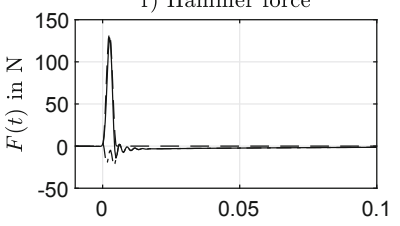

d) Frame acceleration

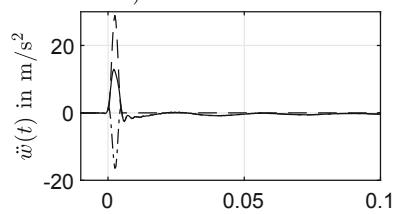

e) Frame acceleration
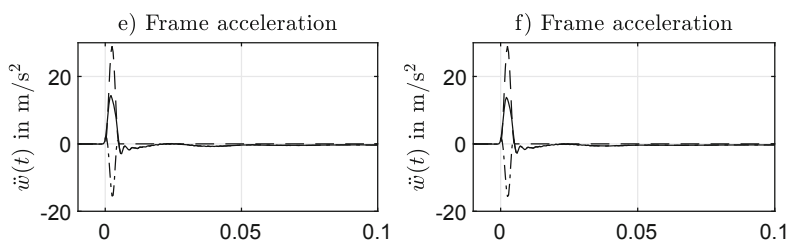

d) Mass acceleration
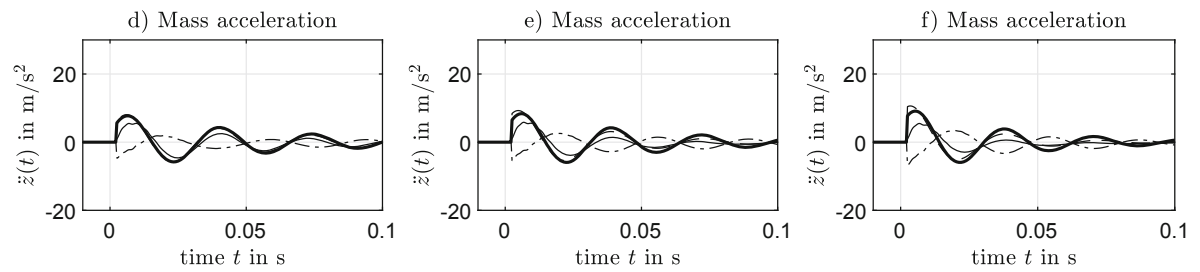

Fig. 5. Outcome, initially guessed from models, from measurement, from calibration, deviations between measurement and initially guessed/calibration of excitation force $F(t)$, velocities and accelerations $\dot{w}(t), \dot{z}(t)$ and $\ddot{w}(t)$ and $\ddot{z}(t)$ of frame and mass in time domain for six damping cases a) to d), Table 2 
calibration results of the stiffness. The authors observed that the higher passive damping force applied by the VCA leads to a shift in the system's eigenfrequency. With increasing gain in the active configuration, the stiffness calibration shows less deviation. After calibration, high deviation in both the calibrated damping coefficient and active gain compared to the initially guessed values remain.

\section{Conclusion}

The active approach for enhanced vibration isolation compared to passive isolation has been proven effective in experimental test and numerical simulation. Further, the investigation shows that with higher passive damping, the prediction of the dynamic outcome via the calibrated analytical model becomes less adequate. In case of data, resp. measurement uncertainty, the deviation of calibrated damping is up to $9 \%$ for highest applied damping, and up to $30 \%$ for stiffness. The stiffness prediction becomes more adequate in cases of active damping with only up to $8 \%$ at highest active damping. In case of model form uncertainty, the active damping cases lead to poor prediction quality of the calibrated passive damping coefficient and of the active gain, up to $50 \%$. One reason is that the prediction by the LSM algorithm does not properly distinguish between the passive and the active model parameters when they are calibrated simultaneously. This problem occurs also when calibrating mass and stiffness properties would be calibrated simultaneously. In both problems, the functional relation in the model between passive and active damping parameters, and between inertia and stiffness, result from ambivalent parameter values within reasonable boundaries. Handling ambivalent calibration parameters is subject to further investigation.

Acknowledgment. This research is funded by the Deutsche Forschungsgemeinschaft (DFG, German Research Foundation) within the Sonderforschungsbereich (SFB, Collaborative Research Center) 805 "Control of Uncertainties in Load-Carrying Structures in Mechanical Engineering" - project number: 57157498.

\section{References}

1. Accel Technologies: Voice Coil Motor/VLR0113-0089-00A, data sheet, High-Tech Industrial Park, No. 1128 East Jiangxing Road, China (2020). www.accel-tec.com. Accessed 6 Nov 2020

2. Der Kiureghian, A., Ditlevsen, O.: Aleatory or epistemic? Does it matter? Struct. Saf. 31(2), 105-112 (2009)

3. Eberhart, R.C., Kennedy, J.: A new optimizer using particle swarm theory. In: Proceedings of 6th International Symposium on Micro Machine and Human Science, pp. 39-43. IEEE, New Brunswick (1995)

4. Farajpour, I., Atamturktur, S.: Error and uncertainty analysis of inexact and imprecise computer models. J. Comput. Civ. Eng. 27(4), 407-418 (2013). https:// doi.org/10.1061/(ASCE)CP.1943-5487.0000233 
5. Higdon, D., Gattiker, J., Williams, B., Rightley, M.: Computer model calibration using high-dimensional output. J. Am. Stat. Assoc. 103(482), 570-583 (2008). https://doi.org/10.1198/016214507000000888

6. Irretier, H.: Basics in Vibration Technology (Grundlagen der Schwingungstechnik), Studium Technik (2000)

7. Kennedy, M.C., O'Hagan, A.: Bayesian calibration of computer models. J. Roy. Stat. Soc. Ser. B (Stat. Methodol.) 63(3), 425-464 (2001). https://doi.org/10.1111/ 1467-9868.00294

8. Lenz, J., Platz, R.: Quantification and evaluation of parameter and model uncertainty for passive and active vibration isolation. In: IMAC-XXXVII A Conference and Exposition on Structural Dynamics, 28-31 January 2020, Orlando, pp. 135-147 (2020). Fl, USA (2019)

9. Li, S., Platz, R.: Observations by evaluating the uncertainty of stress distribution in truss structures based on probabilistic and possibilistic methods. ASME J. Verif. Valid. Uncert. Quantif. 2(3), 031006-031006-9 (2017)

10. Markert, R.: Strukturdynamik (Structural Dynamics), Textbook. Shaker Verlag, Herzogenrath (2013)

11. Melzer, C.M., Platz, R., Melz, T.: Comparison of methodical approaches to describe and evaluate uncertainty in the load-bearing capacity of a truss structure. In: Fourth International Conference on Soft Computing Technology in Civil Engineering, Prague, Czech Republic, 1-4 September 2015

12. PCB Piezotronics, Inc.: ICP Impact Hammer Sensor 086C03, data sheet, 3425 Walden Avenue, Depew, NY, 14043-2495, USA (2010). www.pcb.com

13. PCB Piezotronics, Inc.: ICP Accelerometer 33B52, data sheet, 3425 Walden Avenue, Depew, NY, 14043-2495, USA (2016). www.pcb.com

14. PCB Piezotronics, Inc.: ICP Quarz Force Sensor 208C02, data sheet, 3425 Walden Avenue, Depew, NY, 14043-2495, USA (2019). www.pcb.com

15. Perfetto, S., Rohlfing, J., Infante, F., Mayer, D., Herold, S.: Test rig with active damping control for the simultaneous evaluation of vibration control and energy harvesting via piezoelectric transducers. J. Phys. Conf. Ser. 744 (2016)

16. Platz, R., Ondoua, S., Enss, G.C., Melz, T.: Approach to evaluate uncertainty in passive and active vibration reduction. In: IMAC-XXXII A Conference and Exposition on Structural Dynamics, 3-6 February, Orlando, FL, USA, pp. 345$352(2014)$

17. Platz, R., Enß, G.C.: Comparison of uncertainty in passive and active vibration isolation. In: IMAC-XXXIII A Conference and Exposition on Structural Dynamics, 2-5 February 2015, Orlando, FL, USA, pp. 15-25 (2015)

18. Platz, R., Melzer, C.: Uncertainty quantification for decision making in early design phase for passive and active vibration isolation. In: ISMA 2016 including USD 2016 International Conference on Uncertainty in Structural Dynamics, 19-21 September 2016, Leuven, Belgium, pp. 4501-4513 (2016)

19. Roy, C.J., Oberkampf, W.L.: A complete framework for verification, validation, and uncertainty quantification in scientific computing. Comput. Methods Appl. Mech. Eng. 200(2528), 2131-2144 (2011)

20. Vandepitte, D., Moens, D.: Quantification of uncertain and variable model parameter in non-deterministic analysis. In: IUTAM Symposium on the Vibration Analysis of Structures With Uncertainties, St. Petersburg, Russia, 5-9 July 2009, pp. 15-24 (2009)

21. VDI 2062: Schwingungsisolierung - Vibration Insulation - Insulation elements, Part 2. Beuth Verlag Berlin, Verein Deutscher Ingenieure (2007) 
22. VDI 2064: Aktive Schwingungsisolierung - Active vibration isolation. Beuth Verlag Berlin, Verein Deutscher Ingenieure (2010)

23. Vöth, S.: Dynamik schwingungsfähiger Systeme (Dynamics of vibrations systems). Vieweg \& Sohn verlag, Wiesbaden (2006)

24. Zang, T.A., Hemsch, M.J., Hiburger, M.W., Kenny, S.P., Luckring, J.M., Maghami, P., Padula, S.L., Stroud, W.J.: Needs and opportunities for uncertainty-based multidisciplinary design methods for aerospace vehicles. The NASA STI Program 1, NASA Langley Research Center, Hampton, VA, Technical Report No. NASA/TM-2002-211462 (2002)

Open Access This chapter is licensed under the terms of the Creative Commons Attribution 4.0 International License (http://creativecommons.org/licenses/by/4.0/), which permits use, sharing, adaptation, distribution and reproduction in any medium or format, as long as you give appropriate credit to the original author(s) and the source, provide a link to the Creative Commons license and indicate if changes were made.

The images or other third party material in this chapter are included in the chapter's Creative Commons license, unless indicated otherwise in a credit line to the material. If material is not included in the chapter's Creative Commons license and your intended use is not permitted by statutory regulation or exceeds the permitted use, you will need to obtain permission directly from the copyright holder. 